\title{
The effect of orientation on tactual braille recognition: Optimal touching positions
}

\author{
MORTON A. HELLER \\ Winston-Salem State University, Winston-Salem, North Carolina
}

\begin{abstract}
Subjects in five experiments matched tangible braille against a visible matching code. In Experiment 1, braille recognition suffered when entire lines of braille characters were tilted in varying amounts from the upright. Experiment 2 showed that tilt lowered performance for tangible, large embossed letters, as well as for braille. However, recognition was better for print letters than it was for braille. In Experiment 3, subjects attempted to match the upright array against emboseed braille that was left/right reversed, inverted up/down, or rotated $+180^{\circ}$. Performance was close to that for normal braille in the left/right reversal condition, and very low for the $+180^{\circ}$ rotation group. These results on braille tilt in the "picture plane" may reflect difficulty in manipulating the tangible "image." Braille recognition performance was not lowered when the visible matching array was tilted $-45^{\circ}$ or $-90^{\circ}$ from the upright but the tangible stimuli were upright. In Experiment 4, recognition of left/right reversed braille that was physically horizontal (on the bottom of a shelf) was compared with that of braille left/right reversed due to its location on the back of a panel, in the vertical plane. Braille recognition accuracy was higher with braille located vertically. An additional experiment showed the beneficial effect of locating braille in the vertical, frontoparallel plane, obtained with $+90^{\circ}$ degree rotated braille. It is proposed that optimal tactual performance with tangible arrays might depend on touching position, and on the physical position of stimuli in space. Just as there are good and poor viewing positions, there may be optimal touch. ing positions. The effects of tilt on braille identification were diminished for blind subjects, suggesting the importance of tactile experience and skill.
\end{abstract}

Orientation has been shown to influence the difficulty of tactual braille recognition (Heller, 1987). Upright shapes are generally easier to recognize than tilted shapes (Rock, 1973). Sighted subjects find it very difficult to cope with tilted braille when they are unable to see their hands in relation to the patterns they touch. Blurry sight of the hand helps persons cope with tilt (Heller, 1989b). In addition, tilting the subject's finger degrades recognition of upright braille (Heller, 1987, Experiment 5). It is not known, however, whether visual information about hand orientation is more effective than proprioceptive feedback in aiding braille recognition. Orientation of the patterns relative to the torso and head could also be important. Moreover, information about pattern tilt is critical, and performance suffers when subjects are not told about the nature of the orientation manipulation (Heller, 1986). It is not known whether these results might be found with the use of other sorts of stimuli as well (but see Rock,

Preparation of this report and some of the research were supported by NIH MBRS Grant 2 SO6 RR-08040. I wish to thank Kimberly Nesbitt, Tamala Joyner, Aretha Jones, Katrina Covington, and Keisha Speller for assistance with data collection and analysis. I am grateful to Emerson Foulke for the loan of a special slate that was used to emboss the letters used in Experiment 2. Faith Heller and two anonymous reviewers provided constructive comments on an earlier version of this manuscript. Address reprint requests to Morton A. Heller, Psychology, WinstonSalem State University, Winston-Salem, NC 27110.
1973). The issue of the generalizability of these results arises because orientation has been shown to have little effect on the tactual perception of some punctiform patterns (Warm \& Foulke, 1968). In addition, Warm, Clark, and Foulke (1970) reported that the effects of orientation were linked to stimulus complexity. They found little impact on the tactual recognition of simple shapes, but they did find an effect for complex shapes.

Historically, some researchers have argued that touch is limited in its ability to provide an adequate conception of space and form. Revesz (1950) asserted, for example, that haptic space ignores notions of the vertical and horizontal (see Heller, 1991, p. 15). Furthermore, Revesz claimed that touch only provides a crude understanding of the gross structure of objects, and that it ignores important details. This theoretical viewpoint holds that touch cannot comprehend perspective or covering (but see Heller \& Kennedy, 1990).

The study of orientation in tangible displays is relevant to a current theoretical formulation in haptics. Lederman and Klatzky (1987) have proposed that touch is ill-suited for the perception of two-dimensional configurations. They argue that touch excels in the perception of such substance-related aspects of objects as texture (see Heller, 1989c), thermal attributes, hardness, and so forth. Touch has difficulty with two-dimensional configurations, and tends toward perceptual distortion. According to this view, 
the sense of touch is better suited for determining what a substance is than for determining its spatial arrangement and location in space. Thus, any demonstration of special difficulties for the sense of touch in coping with tilt would be consistent with the Lederman and Klatzky formulation. Although vision is slowed as subjects attempt recognition of tilted patterns, the effects on accuracy are small (Koriat \& Norman, 1985; see also Koriat, Norman, \& Kimchi, 1991). Touch, however, has been shown to be far more sensitive to tilt than vision is, with small amounts of slant leading to substantial decrements in tactual braille recognition (Heller, 1987). It is not known, of course, whether the influence of orientation on tactual pattern perception is due to a lack of familiarity with the use of that sense for pattern recognition. Furthermore, it is possible that the effects of orientation on touch will not generalize to the haptic recognition of tangible patterns that are familiar to sighted subjects, such as print letter shapes. Stimulus characteristics can be important, since there are independent reports that haptic recognition of some raisedline drawings is much easier than that of others (Heller, 1989a; Lederman, Klatzky, Chataway, \& Summers, 1990).

The following experiments were performed to assess the generalizability of the effects of orientation on tactile pattern recognition. In addition, they were designed to reveal factors that might ameliorate the damaging effects of slant. Prior research has shown that visual guidance can help (Heller, 1989b). However, it was considered possible that a number of other factors could alter one's ability to compensate for tilt, including the location of the stimuli in space in relation to the observer (see Oldfield \& Phillips, 1983). This follows from the notion that spatial reference information is important for the sense of touch (Warren, 1984). Experiment 1 was an attempt to replicate earlier findings on orientation, but with the entire array of braille tilted. In Experiment 2, the effect of orientation on braille was compared with that on letters, which are more familiar stimuli. In Experiment 3, subjects were required to compensate for a variety of manipulations of braille, including top/down inversions, left/right reversals, and $+180^{\circ}$ rotations. Subjects in Experiment 4 were exposed to braille that was left/right reversed owing to its location, either behind a vertical panel or on the bottom of a horizontal shelf. In a further experiment, blind subjects attempted braille identification of upright and tilted braille. It was expected that deviations from normal upright stimulus presentations would slow exploration and degrade recognition accuracy.

\section{EXPERIMENT 1 Orientation}

In prior research by the present author, the influence of orientation was evaluated; individual braille characters were tilted, but the braille was arrayed in horizontal lines. This conflict between the orientation of the lines and the braille may have increased task difficulty. Normally, if one reads print material that is tilted, the entire array is slanted, not individual patterns. The subjects in Experiment 1 were asked to make visual matches to braille patterns with the entire array of patterns slanted-a more naturalistic circumstance. It was expected that the effects of orientation would generalize to the tilted array.

\section{Method}

Subjects. The subjects were 84 sighted undergraduates; half were males, and half were females.

Stimuli and Apparatus. The stimuli were standard braille characters, embossed on plastic braille cards with a braille slate. The braille represented the letters B-J. Standard braille uses a $3 \times 2$ matrix of dots and is about $6 \mathrm{~mm}$ high. The three left dots in this matrix are normally designated, top down, as Dots 1,2 , and 3 , while the three on the right are Dots 4,5 , and 6 . Thus, the braille characters representing the letters $A-J$ are constructed from Dot Positions 1, 2, 4 , and 5 . The nine braille patterns, B-J, were presented three times each in a completely random arrangement. The 27 braille characters were arrayed in five lines of 5 characters, with a final line of 2 characters. The tilted lines were inclined at angles of $+15^{\circ},+30^{\circ}$, $+45^{\circ},+60^{\circ},+75^{\circ}$, and $+90^{\circ}$ from the upright. The spacing between the patterns was similar to that in earlier research (Heller, 1987, 1989b).

The braille was fixed on the bottom of a box that was fastened to the front of a large, vertical baffle (see Figure 1). The open front of the box was covered by an opaque, black cloth to eliminate sight of the tangible stimuli, while the open back of the box permitted the experimenter vision of tactual exploration. A drawing of the braille code and letter equivalents was fastened above the opaque top of the exploration box (see Heller, 1987, 1989b, for further details).

Design and Procedure. A simple between-groups design was used. Subjects vocally named the letter that corresponded to the braille pattern that they touched. Tactual exploration was limited to the use of the tip of the index finger of the preferred (writing) hand, and subjects were told to use light pressure. In addition, they were informed about the orientation of the lines of braille, and they were shown a line drawing of the angle of the braille "paper" (this drawing was present throughout). No feedback was given, but subjects were verbally guided back to a line of braille if they became

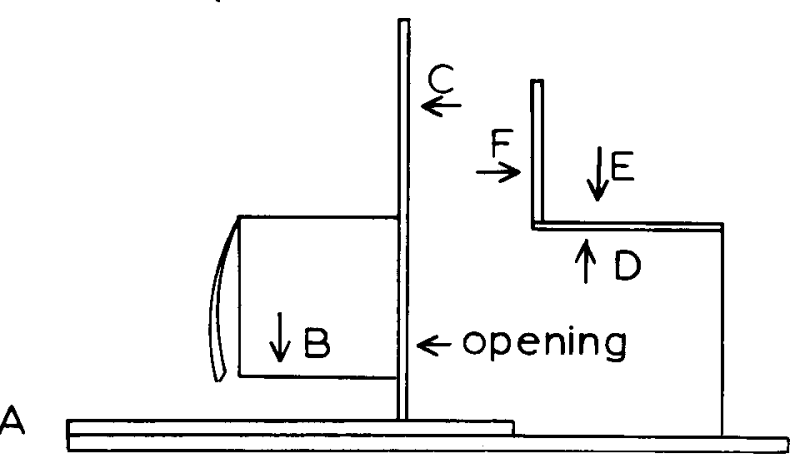

Figure 1. Composite depiction of a side view of the experimental arrangements used in Experiments 1-5. The box on the front of the bafile was present during Experiments 1-3, but was replaced with a cloth covering for the others. The letter $A$ shows the position of the subjects; the letter $B$ indicates the position of the braille during Experiments 1-3. The braille in Experiment 4 was located under a shelf (D) or on the back of a vertical panel (C). In a further experiment, braille was either located on top of a horizontal shelf (E) or placed vertically, on the front of a board ( $F$ ). 
"lost." The subjects in all of the braille experiments reported here were told that they would be timed with a stopwatch on each line of braille, but that they were to try for accuracy.

\section{Results and Discussion}

Performance was degraded by tilt, with as little as $15^{\circ}$ of slant lowering matching accuracy (see Table 1). A oneway analysis (ANOVA) showed a significant effect of orientation on matching accuracy $[F(6,77)=7.4, p<$ $.01]$, and a separate analysis showed an effect on time scores $[F(6,77)=3.29, p<.01]$. Newman-Keuls tests on mean number correct indicated that upright matching scores were higher than all other means, and the mean for $+75^{\circ}$ was lower than the means for braille at $+15^{\circ}$ and $+30^{\circ}$ (all $p \mathrm{~s}<.05$ ). However, all other comparisons failed to reach significance (all $p s>.05$ ). NewmanKeuls tests on mean response latency showed that subjects responded faster to the upright patterns than to those at $+60^{\circ}(p<.05)$. Responses to braille at $+15^{\circ}$ slant were faster than to braille tilted $+60^{\circ}$, but all other comparisons were nonsignificant (all $p s>.05$ ). The results were consistent with earlier reports on the damaging effects of tilt on matching accuracy. It should be noted that prior research has shown that positive and negative slant from the upright produce very similar effects (Heller, 1987).

Experiment 1 showed that the effects of orientation were not specific to the circumstances created by tilting individual patterns in a horizontal array. In Experiment 2, print letters were compared with braille to determine whether orientation also has an impact on familiar patterns.

\section{EXPERIMENT 2 \\ Embossed Letters Versus Braille}

Experiment 2 was designed for the purpose of ascertaining whether orientation would influence tangible letters as much as braille. Letters are more complex than braille, but they are also highly familiar patterns. If the effects of orientation are tied to a lack of familiarity, tilt should have minimal effect on tangible letters.

\section{Method}

Subjects. Seventy-two sighted undergraduates served as subjects ( 33 males and 39 females). None of the subjects had served in Experiment 1.

Stimuli and Apparatus. The braille stimuli were oversized, and represented the letters B-T ( $Q$ was omitted). The print letters and the braille were embossed with the aid of a special slate ( $6 \times 6$ matrix) provided by Emerson Foulke (see Heller, 1989a; Heller \& Kennedy, 1990). Dot size and dot spacing for the letters were similar to those for standard braille. The letters were $1.1 \mathrm{~cm}$ high, as were the braille patterns representing the letters $\mathrm{K}-\mathrm{T}$. The braille characters representing the letters B-J were somewhat smaller $(6 \mathrm{~mm})$, since the patterns were derived from a restricted dot matrix (Dot Positions 1, 2, 4, and 5). Each subject was exposed to two blocks of trials, with the 18 letters randomized within each block, for 36 letters in all. The horizontal spacing between characters was approximately $1.2 \mathrm{~cm}$, and the spacing between rows was about $1.5 \mathrm{~cm}$. Unlike in Experiment 1, the lines were horizontal and the individual patterns were upright or tilted at $+45^{\circ}$ or $+90^{\circ}$ from the upright. The apparatus was the same as in Experiment 1. However, a drawing of the braille code for the letters A-T was present throughout.

Design and Procedure. A two-way, independent-groups design was used. The factors were stimulus type (braille vs. letters) and orientation. Each subject was required to examine the patterns, as in Experiment 1, with the tip of the index finger of the preferred hand. A vocal naming response was required as subjects made visual matches to the tangible code.

\section{Results and Discussion}

Subjects showed an effect of orientation on both letters and braille, but performance was somewhat better for large letters than for braille (see Table 2). A betweengroups ANOVA of number correct showed a significant effect of orientation $[F(2,66)=16, p<.001]$, a significant effect of pattern type $[F(1,66)=11, p=.001]$, and a nonsignificant interaction $(F<1)$. Performance was lower for the $+45^{\circ}$ slant conditions, an oblique effect (Appelle, 1972).

A second ANOVA, performed on mean time scores, showed a significant effect of pattern type, with faster performance for large letters than for braille $[F(1,66)=7.4$, $p<.01]$. Increased slant slowed down the subjects $[F(2,66)=8.2, p<.001]$, but the interaction just failed to reach significance $[F(2,66)=2.9, p=.059]$. Table 2 shows mean time scores per item for embossed letters and braille. The effect of orientation on response latencies seemed somewhat larger for braille (though the interaction was nonsignificant) with very long response latencies for braille at $+90^{\circ}$. It should be noted that an additional ANOVA was performed on the number correct, but with blocks of trials as a third factor. An effect of trials was found, as was an interaction between blocks of trials and orientation (none of the other interactions was significant). The trials effect was limited to slanted stimuli. Thus, subjects became slightly better at coping with tilt as a result of practice.

Table 1

Mean Number Correct and Percent Correct, With Standard Deviations, and Mean Time per Item (in Seconds) as a Function of Orientation (Braille Characters B-J, Entire Page Tilted)

\begin{tabular}{lcrrrrrr}
\hline & \multicolumn{7}{c}{ Orientation } \\
\cline { 2 - 8 } \multicolumn{1}{c}{ Measure } & Upright & $+15^{\circ}$ & $+30^{\circ}$ & $+45^{\circ}$ & $+60^{\circ}$ & $+75^{\circ}$ & $+90^{\circ}$ \\
\hline No. correct & 23.2 & 16.8 & 16 & 13.4 & 10.8 & 8.8 & 14.3 \\
\% correct & 85.6 & 62.3 & 59.3 & 49.7 & 40.1 & 32.4 & 52.8 \\
SD & 3.2 & 5.8 & 7.0 & 6.0 & 4.8 & 5.5 & 6.5 \\
Time/item & 7.1 & 9.9 & 10.8 & 11.9 & 14.7 & 9.9 & 11.3 \\
\hline Note-Maximum score possible $=27$. & & & &
\end{tabular}


Table 2

Mean Number Correct, With Standard Deviations, and Mean Time (in Seconds) for Large Braille and Embossed Print Letters

\begin{tabular}{|c|c|c|c|c|c|c|c|c|c|}
\hline \multirow[b]{4}{*}{ Character } & \multicolumn{9}{|c|}{ Orientation } \\
\hline & \multicolumn{3}{|c|}{ Upright } & \multicolumn{3}{|c|}{$+45^{\circ}$} & \multicolumn{3}{|c|}{$+90^{\circ}$} \\
\hline & \multicolumn{2}{|c|}{ No. Correct } & \multirow[b]{2}{*}{ Time } & \multicolumn{2}{|c|}{ No. Correct } & \multirow[b]{2}{*}{ Time } & \multicolumn{2}{|c|}{ No. Correct } & \multirow[b]{2}{*}{ Time } \\
\hline & $M$ & $S D$ & & $M$ & $S D$ & & $M$ & $\overline{S D}$ & \\
\hline Letters & 29.1 & 5.0 & 12.7 & 19.3 & 5.6 & 16.0 & 24.7 & 6.6 & 17.0 \\
\hline Braille & 25.9 & 6.2 & 13.6 & 13.9 & 6.7 & 19.3 & 17.6 & 9.2 & 29.3 \\
\hline
\end{tabular}

Note-Maximum score possible $=36$.

\section{EXPERIMENT 3 \\ Left/Right Reversals Versus Up/Down Inversion Versus $+180^{\circ}$ Rotation}

In Experiment 3, subjects were exposed to braille that was top/down inverted, left/right reversed, or rotated $+180^{\circ}$ from the upright. The first two conditions pose a problem that could be solved by mental rotations in the third dimension - that is, in depth. The $+180^{\circ}$ rotation entails mental rotation in two-dimensional space-namely, the picture plane. On the assumption that touch is limited in its ability to cope with two-dimensional transformations, one would expect lower performance in the $+180^{\circ}$ condition. ${ }^{1}$

\section{Method}

Subjects. There were 36 subjects (18 males and 18 females), recruited on an undergraduate campus. None of the subjects served in the previous experiments.

Stimuli and Apparatus. The braille stimuli were of standard size and represented the letters B-J. The braille for the left/right reversal condition was embossed with the patterns left/right reversed. Up/down inverted patterns were embossed so that dots that would normally appear in the top two positions of the $3 \times 2$ matrix (Dots 1 and 4) were placed in the middle two positions (Positions 2 and 5 ), and vice versa. The $+180^{\circ}$ rotated braille characters were individually rotated, with lines horizontal. Otherwise, the equipment was identical to that in the earlier experiments. There were, however, 27 recognition trials per subject.

Design and Procedure. In most respects, the procedure was similar to that in the first two experiments. Independent groups of subjects were exposed to braille that was up/down inverted, left/right reversed, or rotated $+180^{\circ}$ from the upright. Subjects were told about the transformation of the patterns and were to engage in a process of mental compensation. That is, subjects were told, for example, that the braille was rotated $+180^{\circ}$, when appropriate, and they were instructed to mentally rotate the impression gained from touching back to the upright. In the $+180^{\circ}$, up/down, and left/right conditions, subjects were shown illustrations of a transformed letter (capital P) to ensure that the instructions were explicit. They were also told that the matching code was normal upright braille. They were to mentally compensate for the transformation of the tangible array and make sure that the impression resulting from touch was "made upright." Thus, subjects in the left/right condition were told that the embossed, tangible patterns were left/right reversed, and they were also told that they were not up/down inverted. As in the earlier experiments, subjects made vocal identifications with the aid of an upright visual matching code. Feedback was not given.

\section{Results and Discussion}

Performance was best when subjects attempted to cope with the left/right reversal. Taken in order, mean num- bers correct for the left/right reversal, up/down rotation, and $+180^{\circ}$ rotation were $19.5,16.8$, and 11.1 (maximum score possible $=27$ ). The effect of stimulus transformation on accuracy was highly significant $[F(2,33)=11.3$, $p<.001]$, but the effect on time scores was nonsignificant $[F(2,33)=1.4, p>.05]$. A Newman-Keuls test on accuracy scores showed that the mean for the $+180^{\circ}$ rotation condition was significantly lower than both other means $(p<.01)$, but the left/right and up/down means were not significantly different $(p>.05)$. It should be noted that subjects performed as well when compensating for left/right reversed braille $(M=19.5)$ as did subjects in a comparable control condition with normal braille $(M=20.1)$ located flat on a shelf $(t<1)$.

There are at least two possible interpretations of the present data. One possibility is that touch has special difficulty in coping with transformations in a two-dimensional configuration (see Lederman \& Klatzky, 1987). The low scores with $+180^{\circ}$ rotations could then represent problems with manipulating tactile images in the "picture plane" (a two-dimensional rotation). This assumes that subjects in the left/right and up/down conditions make a depth rotation to solve the problems posed in the present experiment. An alternative explanation of the data would assume that the $+180^{\circ}$ rotation requires two sorts of imaginal transformations-namely, an up/down inversion and a left/right imaginal reflection. Of course, one cannot rule out propositional sorts of explanations, since they also would predict lower performance in the $+180^{\circ}$ condition. Propositional explanations of the data are also consistent with the nonsignificant effect of the type of rotation on response latency in this experiment.

Experiment 4 was designed to determine whether it is possible to further minimize the damaging effects of rotations on braille. Subjects in Experiment 3 were able to compensate for left/right reversals when instructed appropriately. In Experiment 4, subjects were exposed to left/right reversals, but this was accomplished through manipulation of the location of the stimuli in space.

\section{EXPERIMENT 4 Optimal "Touching Position"}

Oldfield and Phillips (1983) argued that people normally interpret tactile patterns within a larger framework of spatial coordinates. If so, one would expect that braille recognition would be influenced by the position of the braille in space. Braille patterns in Experiment 4 were placed 
under a shelf or on the back of a vertical panel. In both cases, the patterns were left/right reversed with respect to the subjects. Adoption of an egocentric "point of view" would lead to reversal errors under conditions in which subjects were not told about the reversal. One would expect that information about the reversal should lead to fewer reversal errors, and that subjects should be able to adopt an "objective," external frame of reference, a geocentric vantage point. ${ }^{2}$

\section{Method}

Subjects. There were 48 subjects; half were males, and half were females. None served in any of the previous experiments.

Stimuli and Apparatus. The stimuli were standard braille characters, B-J, as in Experiment 3. The stimuli were fastened to the back of the baffle (that was used to obscure vision), above the clothcovered opening. The subjects in the vertical braille conditions had to reach through the opening in the baffle and turn their hands so that their palms were directed toward their faces (with fingers up), since the braille was on the back of the vertical panel. For the horizontal braille condition, the braille paper was fastened beneath a horizontal shelf, about $22 \mathrm{~cm}$ above the table surface (see Figure 1).

Design and Procedure. An independent-groups, two-way design was used. The factors were information about the left/right reversal, and position of the braille in space (vertical or horizontal). Some subjects were told that the patterns would be left/right reversed because of their position in space, and that the matching code was that for normal braille. These subjects were instructed to mentally compensate for the reversal, and that if they did not do this, they would wind up identifying the wrong pattern. A further illustration of the nature of the reversal was provided by showing how a print letter would be reversed by turning a page over, so as to place it on the back or bottom of a surface (as was appropriate). Subjects in the other conditions were simply told to identify the braille by means of the matching code, but were given no information regarding the reversals. It should be pointed out that a couple of these subjects in the no-information, vertical group asked whether they should adopt the point of view of a person on the other side of the baffle. These subjects were instructed: "You decide." As in earlier experiments, subjects scanned each line of braille characters from left to right. Feedback was not given.

It should be noted that the direction in which braille patterns move across the reading finger is reversed when the stimuli are under a shelf. Scanning direction was also reversed in Experiment 3 and with the braille on the back of the vertical panel. Subjects performed at normal levels with left/right reversed braille, given prior information about the reversal in Experiment 3. Thus, changes in tactile scanning direction may not alter performance for sighted subjects, as long as the direction remains constant.

\section{Results and Discussion}

Recognition accuracy was aided by mounting the braille on the rear of a vertical surface (see Table 3). A two-way, independent-groups ANOVA showed that the effect of spatial location (horizontal vs. vertical) was significant $[F(1,44)$ $=6.6, p=.013]$, as was the effect of telling subjects about the reversal $[F(1,44)=50, p<.001]$, but the interaction was nonsignificant $(F<1)$. Given ambiguity, either because of instructions or because of environmental circumstances, subjects will tend to code egocentrically. However, when instructed, subjects were able to adopt a geocentric (exocentric) point of view. Furthermore, the "costs" associated with the vertical position were minimal. A second ANOVA was performed on mean time per item, and none of the effects were significant. If anything, subjects were somewhat faster in the vertical condition.

It should be noted that the results of Experiment 4 are not specific to the inverted palm-up posture. Performance was slightly lower here than with left/right reversals and the palm down in Experiment 3. Thus, it could be argued that the lower performance in the horizontal condition was not due to spatial reference information, to the position of the braille in space, or to the relationship between the exploratory finger and the braille, but was a consequence of an abnormal posture. Consequently, an additional experiment was performed in which two groups of subjects ( $n=10$, total $N=20$ ) examined braille (B-J) that was rotated $+90^{\circ}$ and was either flat on the top of a horizontal shelf, or mounted vertically, on the front of a board that was $22 \mathrm{~cm}$ behind and parallel to the main baffle used in these experiments (in the frontoparallel plane; see Figure 1). Subjects were told about the orientation of the braille. They were significantly more accurate in the vertical condition $(M=17.6)$ than they were when the braille was horizontal, flat on the top of the shelf $[M=12.1$; $t(18)=2.8, p<.05]$. Unfortunately, the subjects took significantly longer when the braille was vertical and was slanted $+90^{\circ}[t(18)=2.3, p<.05]$. This experiment ruled out the possibility that the results of Experiment 4 (the effect of the vertical placement) were simply explicable in terms of an abnormal posture.

How are we to understand the advantages of a vertical array in helping subjects cope with tilt? One possibility is that just as there are optimal viewing positions, there

Table 3

Mean Number Correct, With Standard Deviations, and Mean Exploration Time (in Seconds) for Vertically and Horizontally Mounted Braille (Back of Board or Bottom of Sheln

\begin{tabular}{|c|c|c|c|c|c|c|}
\hline \multirow[b]{4}{*}{ Position } & \multicolumn{6}{|c|}{ Information Condition } \\
\hline & \multicolumn{3}{|c|}{ Told About Reversal } & \multicolumn{3}{|c|}{ Not Told } \\
\hline & \multicolumn{2}{|c|}{ No. Correct } & \multirow[b]{2}{*}{ Time } & \multicolumn{2}{|c|}{ No. Correct } & \multirow[b]{2}{*}{ Time } \\
\hline & $M$ & $S D$ & & $M$ & $S D$ & \\
\hline $\begin{array}{l}\text { Vertical } \\
\text { Horizontal }\end{array}$ & $\begin{array}{l}20.9 \\
17.3\end{array}$ & $\begin{array}{l}5.1 \\
6.1\end{array}$ & $\begin{array}{l}10.6 \\
13.7\end{array}$ & $\begin{array}{r}11.3 \\
8.1\end{array}$ & $\begin{array}{l}4.5 \\
1.5\end{array}$ & $\begin{array}{l}10.2 \\
10.1\end{array}$ \\
\hline
\end{tabular}

Note-Maximum score possible $=27$. 
may be optimal touching positions. ${ }^{3}$ It may not always be best to lay material flat on a desk surface while examining tangible arrays. This explanation is consistent with personal observation of a blind person reading braille notes while giving a talk, who placed his braille cards vertically, in the frontoparallel plane. Additional related explanations will be considered shortly, but it should be noted that the present results may have wide bearing on research on touch, both in the laboratory and in the natural environment. For example, should the results of these experiments generalize to apply to map reading, there would be clear implications for instructing blind persons in their interpretation. It is difficult to read tangible maps when they are misaligned with respect to the environment (Rossano \& Warren, 1989). Perhaps, vertical positioning of maps would help subjects cope with misalignment and coding of spatial information. Also, it is possible that picture recognition might be influenced in a similar manner, and raised-line drawings might be more readily interpreted if "viewers" adopted "better" touching positions. Thus, additional research should examine the effect of positional factors, to ensure that procedures do not underestimate the capability of the sense of touch.

One reviewer wondered whether the results of experiments on touch performed with sighted subjects would apply to experienced braille readers. It is possible, for example, that experience and familiarity with reading braille could reduce the difficulty of coping with pattern tilt. This possibility was also suggested by the trials effect found in Experiment 2, whereby subjects became better at compensating for tilt with practice. Consequently, an experiment was conducted with experienced braille readers to determine whether the effect of pattern tilt would apply to tactually experienced samples. The earlyblind $(n=8)$ and late-blind $(n=8)$ subjects were recruited at Industries of the Blind workshops in WinstonSalem and Greensboro, as well as in the community of Winston-Salem. All identified themselves as fair or good braille readers. The braille consisted of the code for the letters B-J, three times each in a random arrangement. The braille was arrayed in horizontal lines, flat on a horizontal table top, but with the individual patterns upright, tilted $+45^{\circ}$ from the upright, or $+90^{\circ}$ from the upright. Each subject was exposed to the three orientations in random order, with blocks of 27 patterns in each orientation.

A mixed design was employed, with independent groups of early and late blind subjects and repeated measures on orientation. Subjects were exposed to cardboard triangles illustrating the $45^{\circ}$ and $90^{\circ}$ angles. In addition, they were shown an upright ruler and then shown how the ruler could be tilted at each angle. The subjects were informed about the tilt conditions, and they were told to compensate for tilt-that is, they were told to imagine the braille characters as they would appear if upright.

Tilt lowered performance for both groups of blind subjects, but the effect was not as large as it was for sighted subjects restricted to the sense of touch. Table 4 shows performance for the early-blind and late-blind subjects.
Table 4

Mean Number Correct for Early-Blind and Late-Blind Subjects as a Function of Orientation, with Standard Deviations

\begin{tabular}{|c|c|c|c|c|c|c|}
\hline \multirow[b]{3}{*}{ Visual Status } & \multicolumn{6}{|c|}{ No. Correct } \\
\hline & \multicolumn{2}{|c|}{$0^{\circ}$} & \multicolumn{2}{|c|}{$+45^{\circ}$} & \multicolumn{2}{|c|}{$+90^{\circ}$} \\
\hline & $M$ & $S D$ & $M$ & $S D$ & $M$ & $S D$ \\
\hline Early blind $(n=8)$ & 27 & 0 & 15.6 & 6.5 & 17.9 & 5.9 \\
\hline Late blind $(n=8)$ & 27 & 0 & 20.0 & 7.3 & 22.6 & 4.7 \\
\hline All blind $(N=16)$ & 27 & & 17.8 & & 20.3 & \\
\hline Sighted subjects & 27 & 0 & 22.2 & 4.6 & 18.2 & 4.8 \\
\hline
\end{tabular}

Note-Maximum score possibie $=27$. Data for sighted subjects, who viewed tachistoscopically presented braille characters, are from Heller (1987).

In addition, this table shows the results of an earlier experiment conducted with sighted subjects who viewed tachistoscopically projected braille characters (see Heller, 1987). The effect of visual status just failed to reach significance for both accuracy and time scores $(p=.06$ for time, $p=.09$ for accuracy). The effect of orientation was highly significant for accuracy $[F(2,28)=17.3, p<.001]$ and for time $[F(2,28)=12.3, p<.001]$, but all interactions failed to reach significance. Newman-Keuls tests showed that the differences between $45^{\circ}$ and $90^{\circ}$ were nonsignificant. However, I should point out that there are large individual differences in spatial skills. Two of the late-blind subjects showed no errors in any orientation condition. This suggests that the sense of touch may not be as limited as one might expect, given data from naive, sighted, blindfolded subjects.

Furthermore, one cannot help but notice the similarity between the performance of the sighted subjects who used vision in the earlier study (Heller, 1987) and the blind subjects who used touch in the present experiment. Performance with $+90^{\circ}$ tilt was actually slightly higher for the blind subjects (see Table 4). The scores for sighted subjects looking at tilted embossing on manila braille paper were considerably lower (Heller, 1987, Experiment 3). These results suggest that at least part of the difficulty that sighted subjects have in compensating for tilted braille may derive from their relative inexperience and lack of skill in using the sense of touch for pattern perception.

\section{GENERAL DISCUSSION}

The experiments show that orientation has a general effect on tactual pattern recognition, and that this effect is not limited to the recognition of braille. Slant lowered performance for tangible letters in Experiment 2. Subjects can cope with some types of stimulus transformation better than others, and Experiment 3 showed that braille rotated $+180^{\circ}$ was more difficult than up/down or left/right reversed braille. Experiment 4 indicated that when subjects are trying to compensate for left/right reversals, they can be aided by locating braille vertically. An additional experiment on $+90^{\circ}$ tilted braille yielded support for the idea that locating braille in the frontoparallel plane aids the process of compensating for slant. An experiment with 
blind subjects showed that experienced, skilled subjects are also affected by pattern tilt. However, the effect of tilt was diminished for blind readers, suggesting the importance of experience.

The benefits associated with placing braille vertically and in the frontoparallel plane require some comment, since they suggest that there may be optimal touching positions for braille and optimal spatial locations for tangible stimuli. We all take it for granted that viewing conditions are critical for evaluating sight. One would not, for example, conduct an acuity test under low light, unless that were a special interest. The adequacy of visual texture perception may vary with lighting and viewing conditions (see Heller, 1989c). Side lighting maximizes viewing conditions, and flat, diffuse light may impair visual judgments of roughness or smoothness. In addition, one would not normally read a newspaper with the material at eye level, but with the paper sloped away at such an extreme angle as to produce exaggerated foreshortening. Similarly, successful trompe l'oeil art depends on correct viewing position (Gibson, 1966). The results of Experiment $\mathbf{4}$ suggest that all spatial locations may not be equally suitable for the sense of touch, especially when "imaginal manipulations" are called for.

It is necessary to explain why orienting braille in the frontoparallel plane leads to a better touching position. Placing material flat on a table surface prompts tactile scanning behaviors that are sometimes radial to the body and that converge on the individual's torso. Other exploratory movements are then tangential to the body. There is ample evidence that radial movements are overestimated with respect to tangential motions (Day \& Wong, 1971). This radial/tangential effect can produce distorted percepts. Although highly skilled braille readers do not engage in tracing motions, these motions are typical of the novice (see Heller, 1985, 1989b). Naive sighted subjects will normally engage in a process of trying to trace a braille pattern to discover its shape. Thus, naive sighted subjects were able to identify braille by watching other persons touch the braille. These subjects could not see the braille itself, due to the blurring effect of stained glass. They were able, however, to observe the scanning behavior of other subjects, and they could use this information to make visual matches to the braille code (Heller, 1985). These tracing behaviors may persist in relatively unskilled braille readers and have been described as rubbing behaviors. Placing the braille material on a flat surface may produce a distorted tactile impression, due to radial/ tangential effects (see Appelle, 1991). Radial/tangential effects have been used to explain such perceptual distortion as the tactile horizontal/vertical illusion. Here, subjects overestimate a vertical line relative to a horizontal in an inverted $\mathrm{T}$ shape. Placing the tangible stimuli in the frontoparallel plane will eliminate the illusion (see Day \& Avery, 1970; Day \& Wong, 1971).

It is also possible that some of the errors previously generated in studies of tangible displays derive from orienting maps or raised-line pictures horizontally, on a table top (e.g., Heller, 1989a). ${ }^{4}$ Radial/tangential effects are known to occur with $7.5-\mathrm{cm}$ patterns. It is not known, however, whether they occur with finger movements and smaller configurations, or whether they are dependent on arm movements. One might reasonably expect that radial/ tangential effects would be larger, and would generate greater distortion, with tangible pictures or maps than with braille. This follows, since larger patterns will involve substantial arm movements. It is likely that increases in movement extent will magnify radial/tangential effects.

The results of the present study may suggest an optimistic prognosis for the use of touch for pattern information. It is proposed that it might be profitable for researchers to investigate optimal conditions for the use of the sense of touch. They may then discover methods for reducing the perceptual distortion that is frequently discovered. These results do not completely invalidate the theoretical idea that touch generates distorted percepts of two-dimensional configurations. For example, the results of Experiment 3 support this point of view, since rotations in the picture plane were more difficult than up/down and left/right reversals. However, performance in the latter condition was good in Experiment 3 and in Experiment 4, when subjects were informed about the manipulation and stimuli were vertical. Rather, it is suggested that there may be ways to ameliorate some of the difficulties that subjects encounter in interpreting two-dimensional patterns, and thereby to optimize haptics. As it stands, we are unable to eliminate the possibility that lack of education may reduce haptic performance in the examination of tangible pictures, maps, or graphs. The role of experience is suggested by the trials effect reported in Experiment 2. Moreover, the effects of tilt were greatly reduced with blind subjects who were skilled readers of braille. This suggests that we should be wary of attempting to generalize from the sighted to tactually experienced people.

It is difficult to come to any final conclusions about either capabilities or deficiencies in the sense of touch, if we are not clear about optimal touching conditions. This caveat also applies to many studies of intermodal relations, especially those comparing vision and touch, and suggests that one should be cautious in interpreting their results. We will need more information about how haptics functions best if we are to engage in meaningful comparisons with sight. It is clearly inappropriate, and an "unfair" procedure, to compare optimal conditions for vision with poor conditions for haptic exposures.

\section{REFERENCES}

APPELle, S. (1972). Perception and discrimination as a function of stimulus orientation: The "oblique effect" in man and animals. Psychological Bulletin, 78, 266-278.

APPELLE, S. (1991). Haptic perception of form: Activity and stimulus attributes. In M. A. Heller \& W. Schiff (Eds.), The psychology of touch (pp. 169-188). Hillsdale, NJ: Erlbaum.

Corcorun, D. W. J. (1977). The phenomena of the disembodied eye or is it a matter of personal geography? Perception, 6, 247-253.

DAY, R. H., \& AverY, G. C. (1970). Absence of the horizontal-vertical illusion in haptic space. Joumal of Experimental Psychology, 83, 172-173. 
DAY, R. H., \& WoNG, T. S. (1971). Radial and tangential movement directions as determinants of the haptic illusion in an L-figure. Journal of Experimental Psychology, 87, 10-22.

GiBson, J. J. (1966). The senses considered as perceptual systems. Boston: Houghton Mifflin.

Heller, M. A. (1985). Tactual perception of embossed Morse code and Braille: The alliance of vision and touch. Perception, 14, 563-570.

Heller, M. A. (1986). Central and peripheral influences on tactual reading. Perception \& Psychophysics, 39, 197-204.

HeLLER, M. A. (1987). The effect of orientation on visual and tactual braille recognition. Perception, 16, 291-298.

Heller, M. A. (1989a). Picture and pattern perception in the sighted and blind: The advantage of the late blind. Perception, 18, 379-389.

HeLler, M. A. (1989b). Tactile memory in sighted and blind observers: The influence of orientation and rate of presentation. Perception, 18, 121-133.

Heller, M. A. (1989c). Texture perception in sighted and blind observers. Perception \& Psychophysics, 45, 49-54.

Heller, M. A. (1991). Introduction. In M. A. Heller \& W. Schiff (Eds.), The psychology of touch (pp. 1-19). Hillsdale, NJ: Erlbaum.

Heller, M. A., \& Kennedy, J. M. (1990). Perspective taking, pictures, and the blind. Perception \& Psychophysics, 48, 459-466.

KorIat, A., Norman, J. (1985). Mental rotation and visual familiarity. Perception \& Psychophysics, 37, 429-439.

Koriat, A., Norman, J., \& KMCHI, R. (1991). Recognition of rotated letters: Extracting invariance across successive and simultaneous stimuli. Journal of Experimental Psychology: Human Perception \& Performance, 17, 444-457.

LEDERMAN, S. J., \& KLATZKY, R. L. (1987). Hand movements: A window into haptic object recognition. Cognitive Psychology, 19, 342-368.

Lederman, S. J., Kuatzky, R. L., Chataway, C., \& Summers, C. D. (1990). Visual mediation and the haptic recognition of two-dimensional pictures of common objects. Perception \& Psychophysics, 47, 54-64.

LoOmis, J. M., \& Lederman, S. J. (1986). Tactual perception. In K. Boff, L. Kaufman, \& J. P. Thomas (Eds.), Handbook of perception and human performance: Vol. 2. Cognitive processes and performance (pp. 31-1-31-41). New York: Wiley.

OLdFIELD, S. R., \& PHILlips, J. R. (1983). The spatial characteristics of tactile form perception. Perception, 12, 615-626.

Revesz, G. (1950). The psychology and art of the blind. London: Longmans, Green.

Rock, I. (1973). Orientation and form. New York: Academic Press.

Rossano, M. J., W WARREN, D. H. (1989). Misaligned maps lead to predictable errors. Perception, 18, 215-229.

Warm, J. S., Clark, J. L., a Foulke, E. (1970). Effects of differential spatial orientation on tactual pattern recognition. Perceptual \& Motor Skills, 31, 87-94.

WARM, J. S., \& FoULKE, E. (1968). Effects of orientation and redundancy on tactual perception of form. Perceptual \& Motor Skills, 27, 83-89.

W ARREN, D. H. (1984). Blindness and early childhood development. New York: American Foundation for the Blind.

\section{NOTES}

1. The reported difficulty in mentally compensating for tilted braille is not a cognitive deficit specific to braille patterns, since slant lowered performance with letters in Experiment 2 . Thus, the difficulty cannot be explained by a lack of familiarity with braille and seems related to the sense of touch. An experiment was performed to determine whether the effect of slant could be due to problems with a translation mecha- nism across the modalities. The experiment, which was conducted after the suggestion of Patrick Cabe, required subjects to match upright tangible braille against a visual matching code that was tilted at $-45^{\circ}$ or $-90^{\circ}$ from the upright. Subjects were exposed to standard embossed braille representing the letters B-J, three times each in a random arrangement. Visual matching performance did not differ significantly between the two groups of 14 subjects $(t<1)$. Matching accuracy with the code at $-45^{\circ}$ (mean number correct $=21.6, S D=5.1$ ) and at $-90^{\circ}$ (mean $=22.5, S D=3.4$ ) was comparable to performance with standard braille and an upright matching code in prior research (e.g., Heller, 1987 ) and in the present experiments. However, subjects required sig nificantly more time for visual matches with the code set $-90^{\circ}$ from the upright $[t(26)=2.13, p<.05]$.

2. Subjects can readily adopt a geocentric (external) frame of reference, when appropriate (see Heller \& Kennedy, 1990). An experiment was conducted to further study the effects of instructions on coding strategies. The letters $p, q, d$, and $b$ were printed on the foreheads or the backs of the necks of 30 subjects. Independent groups of subjects were told to "name the letter," "name the letter I am drawing," or "take the point of view of the experimenter." It was expected that subjects would be less likely to show reversals due to an egocentric frame of reference if given "objective" instructions designed to minimize ambiguity. Mirror reversals were much less frequent with "objective" (geocentric) instructions, and subjects were far more accurate in identifying the letters from the "experimenter's viewpoint." A number of subjects in the group told to take the point of view of the experimenter reported that they automatically generated a visual image of the letter from an egocentric viewpoint (see Corcoran, 1977) and then engaged in a process of consciously "flipping" the image to make an identification. An increase in mirror reversals on the forehead is not a consequence of poor spatial resolution on that skin surface, since a control experiment showed that mean number of digits correct did not differ significantly between the forehead $(M=16.9$ out of 20$)$ and the back of the neck $(M=18)[t(13)=1.2, p>.05]$. These results are consistent with those of Kubovy, Turock, Best, and Marcus (cited in Loomis $\&$ Lederman, 1986). Kubovy et al. reported that it takes additional time for the subject to adopt vantage points other than the preferred onenamely, that of behind the head, looking forward.

3. The benefit of a vertical display is diminished when "mental manipulation" of a pattern is not required. Twelve subjects attempted braille recognition (B-J, three times in a random arrangement) with the braille fastened to the front of a vertical shelf, in the frontoparallel plane. Performance with the braille in the frontoparallel plane was not significantly better $(M=22.3)$ than that previously reported for normal horizontal braille, flat on a shelf.

4. Visual artists are well aware of the importance of not orienting drawing materials in the horizontal plane. Faith Heller (personal communication) has pointed out that it is very difficult to draw and simultaneously see one's drawing properly, if it is horizontal. Artists' drawing tables normally have provision for angling the table surface so that it is almost vertical. Faith Heller suggested that it may be difficult to see linear perspective properly if the drawing material is flat, on a horizontal surface, while one attempts to depict perspective or draws foreshortened objects. Furthermore, if one looks down at a drawing surface while attempting to depict an object, one is forced to recall the perspective while drawing it. A more nearly vertical table orientation allows one to see the object and drawing at once and facilitates visual comparisons.

(Manuscript received July 10, 1991; revision accepted for publication December 19, 1991.) 